\title{
AZIMUTH-ELEVATION DIRECTION FINDING USING POWER MEASUREMENTS FROM SINGLE ANTENNA
}

\author{
Joni Polili Lie ${ }^{\dagger}$, Thierry Blu ${ }^{\ddagger}$, Chong-Meng Samson See ${ }^{\dagger *}$ \\ †Temasek Laboratories@ NTU, 50 Nanyang Drive, Singapore 637553 \\ *DSO National Laboratories, 20 Science Park Drive, Singapore 118230 \\ ${ }^{\ddagger}$ Dept. of Electronic Engineering, Chinese University of Hong Kong, Shantin, N.T., Hong Kong
}

\begin{abstract}
This paper considers the problem of extending the single antenna power measurements based direction finding to the two-dimensional (2D) case, and proposes a method to estimate the azimuth-elevation direction-of-arrival (DOA) from a matrix of received power. Exploiting the fact that the azimuth-elevation antenna pattern is 2D bandlimited, the problem can be transformed into a 2D spectral analysis problem. The proposed method first decomposes the 2D spectral analysis problem into one-dimensional case and then solved them independently. As the solution does not ensure that the estimated azimuth and elevation is in correct order, the solution is subjected to permutation ambiguity. This can then be solved by finding the permutation that best matches the 2D spectral representation. Simulation results demonstrating the high-resolution capability of the proposed method in two-source case and the effectiveness in fivesource case are also presented in this paper.
\end{abstract}

Index Terms - array signal processing, direction-of-arrival, single antenna direction finding, annihilating filter.

\section{INTRODUCTION}

Multiple source direction finding has been an active research topic for many decades due to its wide application in the fields of radar, sonar, and communications. Conventional and well-known methods are based on MUltiple SIgnal Classification (MUSIC) algorithm [1]. These methods estimate the direction-of-arrival (DOA) from a vector of received signals using an antenna array. Despite its high resolution capability of resolving two-closely spaced sources, the MUSICbased methods are highly sensitive to array model errors [2-4].

Recently, a novel method is introduced for AOA estimation using only single antenna from a vector of power measurements [5]. It exploits the antenna pattern diversity of the receiving antenna that is captured through the received power calculated when the antenna is pointing at different directions. From sampling perspective, capturing the variation in received power across space is similar to spatial sampling sum of Diracs whose locations are the signals' DOA.

In this paper, we consider the extension of the problem to the two-dimensional (2D) case (e.g., azimuth and elevation angle estimation) and propose a method to estimate the azimuth-elevation DOA from a matrix of power measurements as a result of spatial sampling along azimuth-elevation plane.

Utilizing the fact that the azimuth-elevation antenna pattern is $2 \mathrm{D}$ bandlimited, the problem can be transformed into a 2D spectral analysis problem. The proposed method is based on the decomposition of the 2D spectral analysis problem into one-dimensional (1D) case and then solved the azimuth and elevation DOAs independently using the method reported in [5]. As the solution does not guarantee that the estimated azimuth and elevation is in correct order, the solution becomes ambiguous. To solve this permutation problem, we propose to scan over all possible permutations and identified the permutation that best matches the $2 \mathrm{D}$ spectral representation.

\section{BACKGROUND AND SIGNAL MODEL}

Consider a single antenna receiving system, of which the antenna spatial response is non-uniform, receiving the transmission from $K$ sources with its antenna pointing to direction $\tilde{\theta}$.

$$
x(t, \tilde{\theta})=\sum_{k=1}^{K} g\left(\tilde{\theta}-\theta_{k}\right) s_{k}(t)+\eta(t)
$$

where $g\left(\tilde{\theta}-\theta_{k}\right)$ is the antenna attenuation for the signal impinging from $\theta_{k}$ when the orientation of the antenna is at $\tilde{\theta}, s_{k}(t)$ is the $k$-th impinging signal, $\eta(t)$ is the receiver's noise and $K$ is the number of impinging signals.

The received power calculated over a duration $T$, in which $s_{k}(t)$ is assumed to be stationary, can be approximated as

$$
p(\tilde{\theta}) \approx \sum_{k=1}^{K} \underbrace{\left|g\left(\tilde{\theta}-\theta_{k}\right)\right|^{2}}_{a\left(\tilde{\theta}-\theta_{k}\right)} r_{k}+\eta(\tilde{\theta})
$$

where $r_{k}$ and $n(\tilde{\theta})$ denote the power of $k$-th impinging signal and noise, respectively. This approximation holds under the assumption that the impinging signals are uncorrelated.

Let $p_{l} \equiv p\left(\tilde{\theta}_{l}\right)$ denote the received power calculated when the antenna's orientation is at $\tilde{\theta}_{l}$. The problem addressed in [5] is to estimate $\left\{\theta_{k}\right\}_{k=1}^{K}$ given a vector of the received power $\mathbf{p}=\left[p_{1}, \cdots, p_{L}\right]^{T}$. Exploiting the fact that the antenna pattern is bandlimited, the authors in [5] models $a(\theta)$ as a sum finite complex exponentials: $a(\theta)=\sum_{m=-M}^{M} a_{m} e^{j m \theta}$ and transforms the problem into a frequency estimation problem. That is, solving $\left\{y_{-M}, \cdots, y_{M}\right\}$ from the following linear systems of equation:

$$
p_{l}=\sum_{m=-M}^{M} \underbrace{\sum_{k=1}^{K} r_{k} e^{-j m \theta_{k}}}_{y_{m}} a_{m} e^{j m \tilde{\theta}_{l}}+\eta\left(\tilde{\theta}_{l}\right)
$$

Hence, the annihilating filter method can be used to retrieve $\theta_{k}$ from $y_{m}$. In noisy case, the Cadzow denoising is applied prior to the annihilating filter [6]. 
In $2 \mathrm{D}$ case, the received power is defined as a function of the azimuth and elevation angle $(\theta, \gamma)$ and the problem is now extended to estimating the azimuth-elevation DOA $\left\{\theta_{k}, \gamma_{k}\right\}_{k=1}^{K}$ given a $(L \times$ $H$ ) matrix of the received power $\mathbf{P}$ whose elements are defined as

$$
\begin{aligned}
\mathbf{P}_{l, h} & \equiv p\left(\tilde{\theta}_{l}, \tilde{\gamma}_{h}\right) \\
& =\sum_{k=1}^{K} \underbrace{\left|g\left(\tilde{\theta}_{l}-\theta_{k}, \tilde{\gamma}_{h}-\gamma_{k}\right)\right|^{2}}_{a\left(\tilde{\theta}_{l}-\theta_{k}, \tilde{\gamma}_{h}-\gamma_{k}\right)} r_{k}+\eta\left(\tilde{\theta}_{l}, \tilde{\gamma}_{h}\right) .
\end{aligned}
$$

\section{PROPOSED APPROACH}

Utilizing the fact that the antenna pattern is $2 \mathrm{D}$ bandlimited, it can be well approximated as a sum of finite $2 \mathrm{D}$ complex exponentials according to

$$
a(\theta, \gamma)=\sum_{m=-M}^{M} \sum_{n=-N}^{N} a_{m, n} e^{j m \theta} e^{j n \gamma}
$$

where $a_{m, n}$ is the real-valued coefficients that defines the shape of the antenna response. Notice that because $a(\theta, \gamma)$ is a real-valued function, this model is valid if the following is satisfied:

$$
a_{m, n}=a_{m,-n} \quad a_{m, n}=a_{-m, n} \quad \text { and } \quad a_{m, n}=a_{-m,-n}
$$

Also, the antenna pattern is a $2 \mathrm{D} 2 \pi$-periodic non-negative function

$$
a(\theta, \gamma)=a(\theta+2 \pi, \gamma)=a(\theta, \gamma+2 \pi)=a(\theta+2 \pi, \gamma+2 \pi)
$$

and $a(\theta, \gamma) \geq 0, \forall \theta, \gamma$. obtain

Substituting (2) into the received power expression in (1), we

$$
p\left(\tilde{\theta}_{l}, \tilde{\gamma}_{h}\right)=\sum_{k=1}^{K} \sum_{m=-M}^{M} \sum_{n=-N}^{N} r_{k} a_{m, n} e^{j m\left(\tilde{\theta_{l}}-\theta_{k}\right)} e^{j n\left(\tilde{\gamma_{h}}-\gamma_{k}\right)}
$$

For brevity, we ignore the noise expression $\eta\left(\tilde{\theta}_{l}, \tilde{\gamma}_{h}\right)$. With a simple re-arrangement, we arrive at

$$
p\left(\tilde{\theta}_{l}, \tilde{\gamma}_{h}\right)=\sum_{m} \sum_{n} a_{m, n} \sum_{k} r_{k} e^{-j m \theta_{k}} e^{-j n \gamma_{k}} e^{j m \tilde{\theta}_{l}} e^{j n \tilde{\gamma}_{h}}
$$

where $\boldsymbol{\theta}=\left[\theta_{1}, \cdots, \theta_{K}\right]^{T}$ and $\boldsymbol{\gamma}=\left[\gamma_{1}, \cdots, \gamma_{K}\right]^{T}$.

\subsection{Solving for Azimuth and Elevation Angles Independently}

Recall that $p\left(\tilde{\theta}_{l}, \tilde{\gamma}_{h}\right)$ is the element of matrix $\mathbf{P}$. Using the expression in (3), it is possible to formulate $\mathbf{P}$ as a matrix equation in the following form

$$
\mathbf{P}=\boldsymbol{\Phi} \mathbf{Y}(\boldsymbol{\theta}, \gamma) \Psi
$$

where the following matrices' formulation are used: $\mathbf{P}$ is a $(L \times H)$ matrix with its element $\mathbf{P}_{l, h}$ defined in (3); $\boldsymbol{\Phi}$ and $\boldsymbol{\Psi}$ are $(L \times(2 M+$ $1))$ and $((2 N+1) \times H)$ matrices with the element: $\boldsymbol{\Phi}_{l, m}=e^{j m \tilde{\theta_{l}}}$ and $\boldsymbol{\Psi}_{n, h}=e^{j n \tilde{\gamma_{h}}}$, respectively; and $\mathbf{Y}(\boldsymbol{\theta}, \boldsymbol{\gamma})$ is a $((2 M+1) \times$ $(2 N+1))$ matrix with the element $\mathbf{Y}_{m, n}$ defined as

$$
\mathbf{Y}_{m, n}=a_{m, n} \sum_{k} r_{k} e^{-j m \theta_{k}} e^{-j n \gamma_{k}}
$$

The matrix $\mathbf{Y}(\boldsymbol{\theta}, \boldsymbol{\gamma})$ contains the azimuth-elevation DOA information and can be retrieved from the matrix $\mathbf{P}$, given that $\Phi$ and $\Psi$ are known, by solving

$$
\min _{\mathbf{Y}}\|\mathbf{P}-\mathbf{\Phi} \mathbf{Y}(\boldsymbol{\theta}, \gamma) \mathbf{\Psi}\|_{2}^{2}
$$

The solution to this optimization can be expressed as

$$
\widehat{\mathbf{Y}}(\boldsymbol{\theta}, \gamma)=\boldsymbol{\Phi}^{\dagger} \mathbf{P} \boldsymbol{\Psi}^{\dagger}
$$

where the superscript $\dagger$ denotes the pseudo-inverse matrix operation. It is worth noting that the above solution requires that the dimensionality of the power matrix $\mathbf{P}$ satisfies the following condition

$$
L \geq 2 M+1 \text { and } H \geq 2 N+1
$$

Notice that from the expression in (4), $Y_{m, n}$ is a sum of $K$ $2 \mathrm{D}$ complex exponentials weighted by the real-valued coefficients $a_{m, n}$. Although it is possible to solve for the exponent terms in onedimensional (1D) case using spectral analysis technique, solving the 2D case directly as spectral analysis problem is not feasible.

Instead, the azimuth and elevation angle are solved independently. To do so, we propose to decompose $Y_{m, n}$ into two of similar 1D spectral analysis problem. This can be achieved using the following algebraic manipulation. Let $\bar{y}_{m}(\boldsymbol{\theta})$ denote the decomposed $1 \mathrm{D}$ expression associated to the azimuth angle, given by

$$
\begin{aligned}
\bar{y}_{m}(\boldsymbol{\theta}) & =\sum_{n=-N}^{N} \frac{\widehat{\mathbf{Y}}_{m, n}}{a_{m, n}}=\sum_{k=1}^{K} r_{k} e^{-j m \theta_{k}} \sum_{n=-N}^{N} e^{-j n \gamma_{k}} \\
& =\sum_{k=1}^{K} r_{k} \underbrace{\left(1+2 \sum_{n=1}^{N} \cos \left(n \gamma_{k}\right)\right)}_{c_{k}} e^{-j m \theta_{k}}
\end{aligned}
$$

The above formulation is now a typical spectral analysis formulation. It is a sum of $K$ exponentials with the amplitude $r_{k} c_{k}$ and the exponent terms $\theta_{k}$. Thus, the elevation angle $\theta_{k}$ can be solved in the similar way as in 1-D case [5] using the Cadzow-annihilating filter method [6].

Likewise for the elevation angle $\gamma_{k}$, the same algebraic manipulation is used $\bar{y}_{n}(\gamma)=\sum_{m} \widehat{\mathbf{Y}}_{m, n} / a_{m, n}$ and simplified as

$$
\bar{y}_{n}(\boldsymbol{\gamma})=\sum_{k=1}^{K} r_{k}\left(1+\sum_{m=1}^{M} \cos \left(m \theta_{k}\right)\right) e^{-j n \gamma_{k}}
$$

\subsection{Solving Permutation Problem}

At this stage, we have obtained the estimates of $\theta_{k}$ and $\gamma_{k}$ in random order. Without the correct order, these estimates are meaningless. Hence, the problem is to find the permutation that corresponds to the correct match of azimuth-elevation angle. Let $\mathcal{S}=$ $\left\{s_{1}, s_{2}, \cdots, s_{K}\right\}$ denote the set that contains all the possible permutations of $\left\{\gamma_{1}, \gamma_{2}, \cdots, \gamma_{K}\right\}$. Because the cardinality of $\mathcal{S}$ is finite $(|\mathcal{S}|=K !)$, it is possible to scan through all the permutations in $\mathcal{S}$ and find the one that minimizes the $\ell_{2}$-norm of the error matrix

$$
\xi\left(s_{z}, \boldsymbol{\theta}, \boldsymbol{\gamma}\right)=\left\|\frac{1}{a_{m, n}}\left(\widehat{\mathbf{Y}}_{m, n}-a_{m, n} \sum_{k=1}^{K} e^{-j m \hat{\theta}_{k}} e^{-j n \hat{\gamma}_{s_{z}(k)}}\right)\right\|_{2}
$$

where $\|\cdot\|_{2}$ denotes the matrix $\ell_{2}$-norm and the subscript $s_{z}(k)$ in $\hat{\gamma}_{s_{z}(k)}$ determines the order of the estimated elevation DOA to be matched with $\hat{\theta}_{k}$. For illustration, consider $K=3$ and if $s_{z}=$ $\{2,1,3\}$, then $\left\{\hat{\gamma}_{s_{z}(k)}\right\}_{k=1}^{K}=\left\{\hat{\gamma}_{2}, \hat{\gamma}_{1}, \hat{\gamma}_{3}\right\}$. Scanning for the minimum $\xi\left(s_{z}, \boldsymbol{\theta}, \gamma\right)$ can be expressed mathematically as follows

$$
s_{\text {opt }}=\arg \min _{s_{z} \in \mathcal{S}} \xi\left(s_{z}, \boldsymbol{\theta}, \boldsymbol{\gamma}\right)
$$

Finally, given $s_{\text {opt }}$ obtained from solving (8), the estimated azimuth-elevation DOAs are

$$
\left\{\left(\hat{\theta}_{1}, \hat{\gamma}_{s_{o p t}(1)}\right),\left(\hat{\theta}_{2}, \hat{\gamma}_{s_{o p t}(2)}\right), \cdots,\left(\hat{\theta}_{K}, \hat{\gamma}_{s_{\text {opt }}(K)}\right)\right\}
$$




\subsection{On Sampling for Multidimensional FRI signals}

Note that the solution proposed here is also applicable generally to sampling of multi-dimensional signals with finite rate of innovation (FRI). An earlier attempt to solve the problem is reported in [7] and the authors proposed a method that requires B-spline kernel as the sampling kernel. On the contrary, our approach can be employed to arbitrary kernel that satisfies the bandlimited property.

Besides solving for the location of the Diracs, a typical FRI sampling [8] requires the amplitude of them to be recovered as well. Likewise for the multidimensional sampling problem, we need to calculate $\hat{r}_{k}$ from the expression in (6). Let $\psi_{k}$ denote the $k$-th coefficient of the exponentials (6). Given $\left\{\left(\hat{\theta}_{k}, \hat{\gamma}_{k}\right)\right\}_{k=1}^{K}$ and $s_{\text {opt }}, \hat{\psi}_{k}$ can be calculated as the over-determined solution of the following linear matrix equation

$$
\left[\begin{array}{c}
\bar{y}_{-M}^{d} \\
\bar{y}_{-M+1}^{d} \\
\vdots \\
\bar{y}_{M}^{d}
\end{array}\right]=\left[\begin{array}{ccc}
u_{1}^{-M} & \cdots & u_{K}^{-M} \\
u_{1}^{-M+1} & \cdots & u_{K}^{-M+1} \\
\vdots & \ddots & \vdots \\
u_{1}^{M} & \cdots & u_{K}^{M}
\end{array}\right] \boldsymbol{\psi}
$$

where $\boldsymbol{\psi}=\left[\psi_{1}, \cdots, \psi_{K}\right]^{T}, u_{k}=e^{-j \hat{\theta}_{k}}$ and the superscript $d$ represents $\bar{y}_{m}(\boldsymbol{\theta})$ after Cadzow de-noising [9]. Then, $\hat{r}_{k}$ can be calculated using the following relationship

$$
\hat{r}_{k}=\hat{\psi}_{k} /\left(\hat{c}_{s_{\text {opt }}(k)}(\gamma)\right)
$$

\subsection{Algorithm Summary}

To summarize the calculations proposed in this paper, a step-by-step outline of the proposed algorithm is listed here

1. Calculate $\mathbf{Y}(\boldsymbol{\theta}, \boldsymbol{\gamma})$ from the power matrix $\mathbf{P}$ according to (5).

2. Decompose into azimuth- and elevation- only spectral formulation, as given by (6), (7).

3. Solve independently $\hat{\theta}_{k}$ and $\hat{\gamma}_{k}$ from $\bar{y}_{m}(\boldsymbol{\theta})$ and $\bar{y}_{n}(\boldsymbol{\gamma})$, respectively. This requires the Cadzow de-noising and annihilating filter method described in [5].

4. Correct the order of $\hat{\gamma}_{k}$ according to $s_{\text {opt }}$ obtained from (8).

5. The final azimuth-elevation DOA estimates are given by (9).

\section{SIMULATION RESULTS}

In this section, we consider a directional antenna with the azimuthelevation antenna pattern simulated using the expression in (2) with $M=N=3$ and the real-valued coefficients $\left\{a_{m, n}\right.$ generated randomly according to uniform distribution. Then, the $a_{0,0}$ is set such that the antenna pattern is a nonnegative function. Fig. 1 shows an example of the azimuth-elevation antenna pattern.

Consider two uncorrelated sources emitting from azimuthelevation $\left(\theta_{k}, \gamma_{k}\right):\left(92.5790^{\circ}, 85.2080^{\circ}\right)$ and $\left(47.7191^{\circ}, 53.5891^{\circ}\right)$. Without loss of generality, the signals received at the antenna are assumed to be modeled as single-tone:

$$
s_{k}\left(t_{n}\right)=\sqrt{\alpha_{k}} \exp \left(j\left[2 \pi f_{k} t_{n}+\varphi_{k}\right]\right)
$$

with the parameters set as: $\alpha_{k}=[0.9,0.8], f_{k}=[0.253,0.347]$, and $\varphi_{k}=[0.0136,0.8044]$. The noise is modeled as complex white Gaussian noise: $\eta\left(t_{n}\right)=\mathcal{N}\left(0, \sigma^{2}\right)+j \mathcal{N}\left(0, \sigma^{2}\right)$ where $\sigma^{2}$ is calculated such that $\mathrm{SNR} \equiv \sum_{k} \alpha_{k} /\left(2 \sigma^{2}\right)$ is $10 \mathrm{~dB}$. As many as 512 samples of the received signal are used to compute the received power

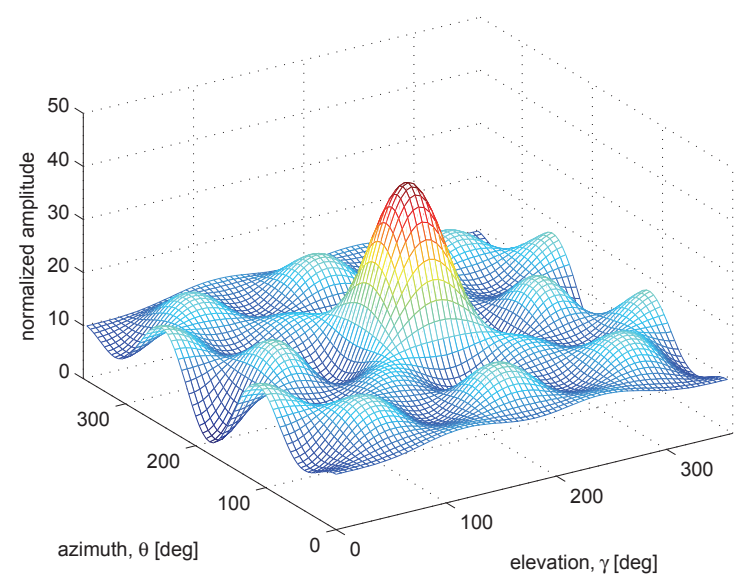

Fig. 1. An example of the realization of azimuth-elevation antenna pattern simulated according to (2) with $M=N=3$.

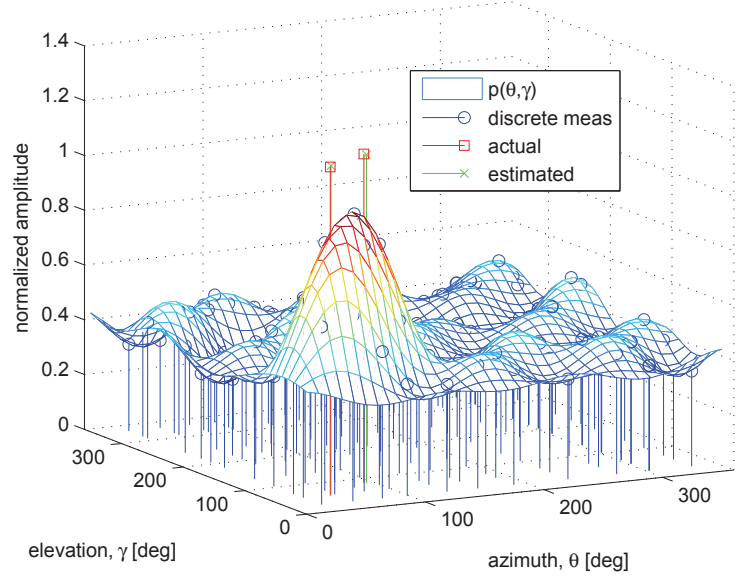

Fig. 2. An example of the received power measurements collected and the location of the actual and estimated azimuth-elevation DOA.

and $(L \times H)=(12 \times 12)$ samples of the received power $p\left(\tilde{\theta}_{l}, \tilde{\gamma}_{h}\right)$ are collected from different azimuth-elevation antenna orientation generated randomly within $0^{\circ}$ and $359^{\circ}$. The mesh plot in Fig. 2 shows the illustration of the continuous received power as a function of the azimuth-elevation orientation of the antenna, $p(\tilde{\theta}, \tilde{\gamma})$ while the stem plot in the same figure shows the discrete $(12 \times 12)$ grid at which the received power measurements are collected in order to form the power matrix $\mathbf{P}$ in the noiseless case. Using the proposed method summarized in Sec. 3.4, the estimated azimuth-elevation DOA are compared with the actual one as shown in Fig. 2. From these results, it is evident that the proposed method belongs to the high-resolution DF method due to its ability to resolve two signal sources that are separated less than a beamwidth apart [10].

To demonstrate the efficacy of the algorithm in more-than-two sources case, we consider $K=5$ with actual azimuth-elevation DOAs: $\left(\theta_{k}, \gamma_{k}\right):\left(92.5790^{\circ}, 85.2080^{\circ}\right),\left(47.7191^{\circ}, 53.5891^{\circ}\right)$, $\left(314.0214^{\circ}, 124.2315^{\circ}\right),\left(154.1285^{\circ}, 224.125^{\circ}\right)$ and $\left(247.825^{\circ}\right.$, $148.2725^{\circ}$ ). To generate the received signals, we use the previous model with the following parameters: $\alpha_{k}=[0.9,0.8,1,0.7,0.85]$, 
$f_{k}=[0.253,0.377,0.1452,0.465,0.3054], \varphi_{k}=[0.0136,0.8044$, $0.2123,0.6872,0.5340]$. The azimuth-elevation antenna pattern is also simulated using the previous model with $M=N=6$ and $(20 \times 20)$ power measurements are collected when the azimuthelevation orientation of the antenna is randomly set.

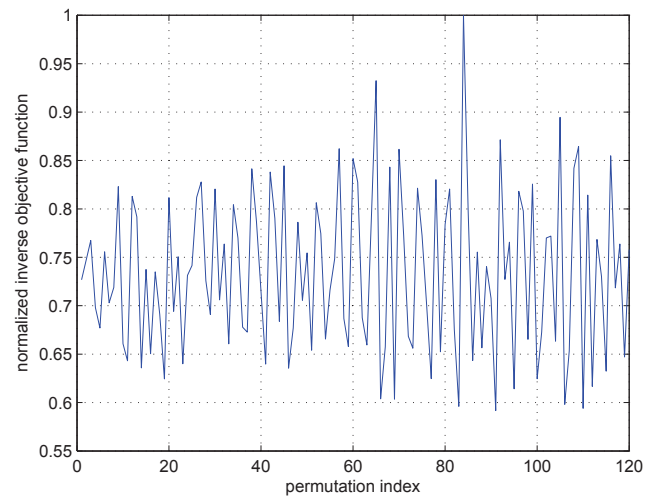

Fig. 3. Normalized inverse objective function in (8) plotted as a function of the permutation index.

Unlike the two-source case, the number of possible permutation has significantly increased. Fig. 3 shows the normalized inverse objective function $\xi\left(s_{z}, \boldsymbol{\theta}, \boldsymbol{\gamma}\right)$, plotted as a function of the permutation index $z=\{1,2 \cdots, K !\}$. It shows that the correct permutation can be obtained from the minimum objective function. As a result, the correct combination of azimuth-elevation estimates matches the actual azimuth-elevation DOAs. This is shown in Fig. 4.

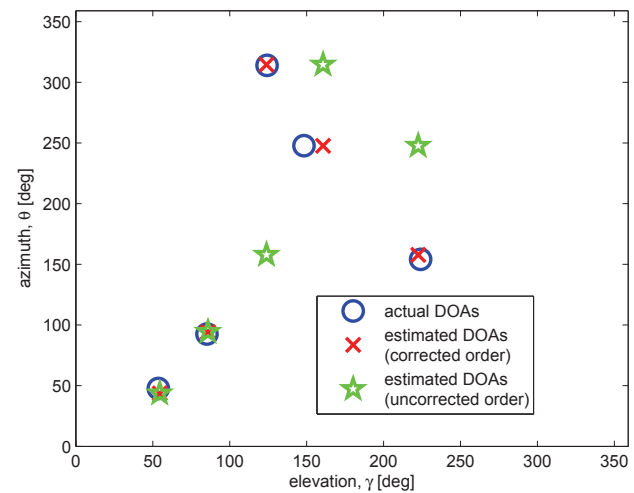

Fig. 4. Actual and estimated azimuth-elevation DOAs.

Lastly, we consider 1000 Monte-Carlo realizations for twosource case and calculate the root mean-square error (RMSE) from the corresponding 1000 azimuth and elevation DOA estimation results independently. These results obtained across different SNR values are then plotted in Fig. 5. From this Figure, it can be observed that the performance improves as the SNR increases.

\section{CONCLUSSION}

This paper proposes the azimuth-elevation DOA estimation method using power measurements from single antenna. As the antenna pat-

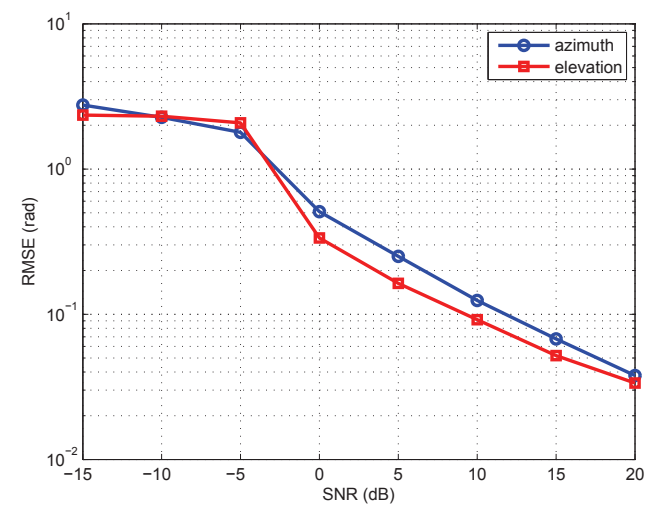

Fig. 5. DOA estimation RMSEs versus SNR for two-source case.

tern is $2 \mathrm{D}$ bandlimited, the problem can be transformed into a $2 \mathrm{D}$ spectral analysis problem. The proposed method is based on the decomposition of the 2D spectral analysis into 1D expression, in which the azimuth and elevation DOAs can be solved independently using the Cadzow-annihilating filter method. Since the Cadzowannihilating filter method does not guarantee that the estimated azimuth and elevation is in correct order, the solution becomes ambiguous. It is also shown that this ambiguity problem can be solved by identifying the permutation that best matches the $2 \mathrm{D}$ spectral expression.

\section{REFERENCES}

[1] R. Schmidt, "Multiple emitter location and signal parameter estimation,” IEEE Trans. Antennas Propagat., vol. 34, no. 3, pp. 276-280, Mar. 1986.

[2] M. Kaveh and A. Barabell, "The statistical performance of the music and the minimum-norm algorithms in resolving plane waves in noise," IEEE Trans. Acoust., Speech, Signal Processing, vol. 34, no. 2, pp. 331-341, Apr. 1986.

[3] B. Friedlander and A. J. Weiss, "Eigenstructure methods for direction finding with sensor gain and phase uncertainties," in Proc. ICASSP-88, Apr. 11-14, 1988, pp. 2681-2684.

[4] P. Stoica and A. Nehorai, "Music, maximum likelihood, and cramer-rao bound: further results and comparisons," IEEE Trans. Acoust., Speech, Signal Processing, vol. 38, no. 12, pp. 2140-2150, Dec. 1990.

[5] J. P. Lie, T. Blu, and C. M. S. See, "Single antenna power measurements based direction finding," IEEE Trans. Signal Processing, vol. 58, no. 11 , pp. 5682-5692, 2010 .

[6] T. Blu, P.-L. Dragotti, M. Vetterli, P. Marziliano, and L. Coulot, "Sparse sampling of signal innovations," IEEE Signal Processing Magazine, vol. 25 , no. 2, pp. 31-40, 2008.

[7] P. Shukla and P. Dragotti, "Sampling schemes for multidimensional signals with finite rate of innovation," IEEE Trans. Signal Processing, vol. 55 , no. 7 , pp. $3670-3686$, july 2007.

[8] M. Vetterli, P. Marziliano, and T. Blu, "Sampling signals with finite rate of innovation," IEEE Trans. Signal Processing, vol. 50, no. 6, pp. 1417-1428, Jun. 2002.

[9] J. A. Cadzow, "Signal enhancement-a composite property mapping algorithm," IEEE Trans. Acoust., Speech, Signal Processing, vol. 36, no. 1, pp. 49-62, Jan. 1988.

[10] H. Krim and M. Viberg, "Two decades of array signal processing research: the parametric approach," IEEE Signal Processing Mag. vol. 13, no. 4, pp. 67-94, Jul. 1996. 